
J.H. Lichtman, $\mathrm{PhD}$
S.B. Jones, MPH
Y. Wang, PhD
E. Watanabe, MPH
E. Leifheit-Limson, $\mathrm{PhD}$
L.B. Goldstein, MD

Address correspondence and reprint requests to Dr. Judith $\mathrm{H}$. Lichtman, Yale University School of Medicine, PO Box 208034

New Haven, CT 06520

judith.lichtman@yale.edu

\title{
Outcomes after ischemic stroke for hospitals with and without Joint Commission-certified primary stroke centers
}

\section{ABSTRACT}

Background: The Joint Commission (JC) began certifying primary stroke centers (PSCs) in the United States in 2003. We assessed whether 30-day risk-standardized mortality (RSMR) and readmission (RSRR) rates differed between hospitals with and without JC-certified PSCs in 2006.

Methods: The study cohort included all fee-for-service Medicare beneficiaries $\geq 65$ years old discharged with a primary diagnosis of ischemic stroke (International Classification of Diseases, ninth revision, Clinical Modification 433, 434, 436) in 2006. Hierarchical linear regression models calculated hospital-level RSMRs and RSRRs, adjusting for patient demographics, comorbid conditions, and hospital referral region. Hospitals were categorized as being higher than, no different from, or lower than the national average.

Results: There were 310,381 ischemic stroke discharges from 315 JC-certified PSC and 4,231 noncertified hospitals. Mean overall 30-day RSMR and RSRR were 10.9\% $\pm 1.7 \%$ and $12.5 \% \pm$ $1.4 \%$, respectively. The RSMRs of hospitals with JC-certified PSCs were lower than in noncertified hospitals $(10.7 \% \pm 1.7 \%$ vs $11.0 \% \pm 1.7 \%)$, but the RSRRs were comparable $(12.5 \% \pm 1.3 \%$ vs $12.4 \% \pm 1.7 \%)$. Almost half of JC-certified PSC hospitals had RSMRs lower than the national average compared with $19 \%$ of noncertified hospitals, but $13 \%$ of JC-certified PSC hospitals had lower RSRRs vs 15\% of noncertified hospitals.

Conclusions: Hospitals with JC-certified PSCs had lower RSMRs compared with noncertified hospitals in 2006; however, differences were small. Readmission rates were similar between the 2 groups. PSC certification generally identified better-performing hospitals for mortality outcomes, but some hospitals with certified PSCs may have high RSMRs and RSRRs whereas some hospitals without PSCs have low rates. Unmeasured factors may contribute to this heterogeneity. Neurology ${ }^{\circledR} 2011 ; 76: 1976-1982$

\section{GLOSSARY}

CMS = Centers for Medicare \& Medicaid Services; FFS = fee-for-service; HRR = hospital referral region; ICD-9-CM = International Classification of Diseases, ninth revision, Clinical Modification; JC = Joint Commission; NQF = National Quality Forum; PSC = primary stroke center; RSMR = risk-standardized mortality rate; RSRR = risk-standardized readmission rate.

The Joint Commission (JC) began certifying primary stroke centers (PSCs) in the United States in 2003. ${ }^{1-4}$ Studies assessing the impact of PSC certification have focused on process measures $^{5-8}$; information on outcomes is limited. One study found hospitals receiving JC PSC certification within the first few years of the program had lower 30-day mortality and readmission rates than hospitals without certified centers before the program began, suggesting that they already used processes that led to better stroke outcomes. ${ }^{9}$ Whether JC PSC certification per se results in better patient outcomes or simply identifies hospitals with better performance is uncertain.

Risk-standardized mortality and readmission after hospital discharge are being used as indicators of the quality of hospital-level care for several disorders, including acute myocardial 
infarction and heart failure. ${ }^{10-13}$ In 2008, the Centers for Medicare \& Medicaid Services (CMS) began publicly reporting hospitallevel risk-standardized 30-day mortality and readmission rates for these conditions. ${ }^{14-18} \mathrm{Al}$ though similar measures are not currently available for stroke, ${ }^{19}$ such measures are planned. High risk-standardized mortality rates (RSMRs) and risk-standardized readmission rates (RSRRs) may indicate poorer quality of care, unresolved problems at initial discharge, the quality of immediate posthospital care, a more chronically ill population, or combinations of these factors. ${ }^{14}$ High readmission rates are also associated with substantial economic burden, and the reduction of avoidable readmissions is an important US health care reform goal. ${ }^{14,15}$

To assess the impact of JC-certified PSCs on stroke outcomes, we determined hospitallevel RSMRs and RSRRs for ischemic stroke discharges from all US hospitals in 2006.

METHODS Cohort. The study population included all Medicare fee-for-service (FFS) beneficiaries 65 years of age or older hospitalized with a primary discharge diagnosis of ischemic stroke (International Classification of Diseases, ninth revision, Clinical Modification [ICD-9-CM] codes 433, 434, and 436) from January 1-December 31, 2006. Data were obtained from the Medicare Provider Analysis and Review files that included demographic information and primary and secondary discharge diagnosis codes for all hospitalizations. Patients who were younger than 65 years were not included in the analysis because they do not represent typical Medicare patients. Patients who were discharged from nonacute care facilities, transferred to or from another acute care facility, discharged within 1 day of admission, or who left the hospital against medical advice were excluded. We included patients with 12 months of continuous Medicare FFS enrollment before and 1 month after the hospitalization to obtain complete medical history, mortality, and readmission information. Hospitals were classified as to whether or not they had a JC-certified PSC. We identified 315 JC-certified PSCs from the start of the certification program in November 2003 through May 30, 2007, by matching the Medicare provider numbers with an online list of JC-certified centers available in May $2007 .^{3}$

Outcomes. Study outcome measures included 30-day allcause RSMRs and 30-day all-cause RSRRs. Mortality was assessed from the date of hospital admission using the Medicare Enrollment Database. The accuracy of ascertainment of vital status using these data resources is high for this age group. ${ }^{20}$ Readmissions included those for any cause to acute care hospitals treating Medicare patients occurring within 30 days of discharge. We excluded readmissions for procedures that may represent planned continuation of treatment after discharge from the index stroke admission, unless acute stroke (ICD9-CM 433.x1, 434.x1) was listed as the principal discharge diagnosis for the hospital readmission. Planned readmissions included carotid endarterectomy, carotid stenting, percutaneous carotid stenting, intercranial and intervertebral stenting, patent foramen ovale closure, ablation, aortic or mitral valve replacement, and cranioplasty.

Covariates. Patient comorbidities were identified using the primary and 9 secondary codes from claims submitted in the year before the index hospitalization and from claims found in the index admission for those conditions that could not represent a complication of the admission. A total of 29 independent variables were included from inpatient administrative claims data, including 2 demographic variables (age and sex), 7 cardiovascular and stroke history variables, and 20 other variables that identify additional coexisting illnesses. The majority of these variables were included in the validated CMS acute myocardial infarction and heart failure 30-day all-cause hospital-specific mortality and readmission measures. ${ }^{10-13}$

Standard protocol approvals, registrations, and patient consents. The Centers for Medicare \& Medicaid Services reviewed and approved the submission of the manuscript, based on data use only. The Human Investigation Committee at Yale University determined that Institutional Review Board approval was not required for this analysis.

Statistical analysis. Bivariate analyses were used to compare patient characteristics and outcomes between JC-certified PSC and noncertified hospitals. $\chi^{2}$ tests were used for comparing binary variables and Wilcoxon rank sum tests were used for comparing continuous variables. Consistent with methods described in the development of administrative models, ${ }^{21,22}$ we estimated a risk model relating the log-odds of 30-day mortality to patient risk factors for the study cohort. The model provided data to compute standardized hospital-specific estimates as well as quantitative summaries of between-hospital variation after adjusting for case mix. An RSMR was calculated for each hospital using the regression coefficients from the risk model. Because of the need to correct for within-hospital clustering of patients and varying hospital volumes, an adjusted number of observed mortalities in the ratio was used rather than the observed number. Geographic differences were accounted for by classifying hospitals into 305 hospital referral regions (HRRs) based on their locations, and then calculating HRR-specific aggregated weighted RSMRs. The same analytic methods were used to calculate RSRRs, with additional censoring for in-hospital deaths and deaths up to 30 days after discharge. Patient mortality and readmission models were risk-adjusted for age, sex, and comorbid conditions consistent with risk-standardization measures developed for the CMS and endorsed by the National Quality Forum (NQF) for hospital performance evaluation..$^{10,12,13}$ The hospital classification (RSMR/RSRR higher than, same as, and lower than the national average) was based on comparing the 95\% confidence intervals with the national average.

The analyses were repeated restricting the ischemic stroke cohort to ICD-9-CM 433.x1, 434.x1, and 436, as these additional modifier codes increase specificity to identify acute ischemic events. ${ }^{23,24}$ All statistical testing was 2-sided, at a significance level of $\alpha=0.05$. Hierarchical models were estimated using the GLIMMIX procedure in SAS. All analyses were conducted using SAS version 9.2 (SAS Institute Inc., Cary, NC) and Stata 9.0 (StataCorp, College Station, TX).

RESULTS There were 310,381 ischemic stroke discharges from the 315 JC-certified PSC hospitals and 


\begin{tabular}{|c|c|c|c|c|}
\hline Table & \multicolumn{4}{|c|}{$\begin{array}{l}\text { Patient characteristics for discharges from hospitals with and without } \\
\text { JC-certified primary stroke centers }\end{array}$} \\
\hline Charact & istic & $\begin{array}{l}\text { Noncertified, } \\
\text { mean } \pm \text { SD or \% }\end{array}$ & $\begin{array}{l}\text { JC-certified PSC, } \\
\text { mean } \pm \text { SD or } \%\end{array}$ & $\begin{array}{l}p \text { Value for } \\
\text { difference }\end{array}$ \\
\hline Hospita & scharges, total n & 246,926 & 63,455 & \\
\hline Age, $y$ & & $78.6 \pm 7.8$ & $78.1 \pm 7.7$ & $<0.0001$ \\
\hline Male se & & 44.7 & 47.9 & $<0.0001$ \\
\hline \multicolumn{5}{|c|}{ Comorbid conditions } \\
\hline \multicolumn{2}{|c|}{ Cerebrovascular disease } & 34.9 & 40.7 & $<0.0001$ \\
\hline \multicolumn{2}{|c|}{ Hypertension } & 64.8 & 65.6 & 0.0002 \\
\hline \multicolumn{2}{|c|}{ Diabetes } & 28.0 & 27.0 & $<0.0001$ \\
\hline \multicolumn{2}{|c|}{ Renal failure } & 11.3 & 12.2 & $<0.0001$ \\
\hline \multicolumn{2}{|c|}{$\begin{array}{l}\text { Chronic obstructive } \\
\text { pulmonary disease }\end{array}$} & 15.4 & 14.4 & $<0.0001$ \\
\hline \multicolumn{2}{|c|}{ Congestive heart failure } & 16.9 & 16.5 & 0.0081 \\
\hline \multicolumn{2}{|c|}{$\begin{array}{l}\text { Acute myocardial } \\
\text { infarction }\end{array}$} & 1.4 & 1.6 & 0.0003 \\
\hline \multicolumn{2}{|c|}{$\begin{array}{l}\text { Peripheral vascular } \\
\text { disease }\end{array}$} & 10.4 & 11.7 & $<0.0001$ \\
\hline \multicolumn{2}{|c|}{$\begin{array}{l}\text { Cardiopulmonary-respiratory } \\
\text { failure }\end{array}$} & 3.6 & 4.5 & $<0.0001$ \\
\hline \multicolumn{2}{|c|}{ Unstable angina } & 0.5 & 0.5 & 0.0155 \\
\hline \multicolumn{2}{|c|}{ Pneumonia } & 6.3 & 5.7 & $<0.0001$ \\
\hline \multicolumn{2}{|c|}{ Dementia } & 12.4 & 10.4 & $<0.0001$ \\
\hline \multicolumn{2}{|c|}{ Depression } & 5.0 & 5.0 & 0.6241 \\
\hline \multicolumn{2}{|c|}{ In-hospital death } & 4.7 & 4.4 & 0.0014 \\
\hline \multicolumn{2}{|c|}{$\begin{array}{l}\text { Discharge to skilled nursing } \\
\text { facility/intermediate care } \\
\text { facility }\end{array}$} & 21.5 & 18.6 & $<0.0001$ \\
\hline \multicolumn{2}{|c|}{ Length of stay, $d$} & $4.6 \pm 4.4$ & $4.5 \pm 4.7$ & $<0.0001$ \\
\hline
\end{tabular}

Abbreviations: $\mathrm{JC}=$ Joint Commission; PSC $=$ primary stroke center.

4,197 noncertified hospitals in 2006 (table). Patients treated at hospitals with and without JC-certified PSCs were of similar age and had comparable rates of comorbid conditions, but JC-certified PSC hospitals had a higher percentage of men and patients with prior cerebrovascular disease. Hospitals with JCcertified PSCs had slightly lower in-hospital mortality rates $(4.4 \%$ vs $4.7 \%)$ and had a lower percentage of patients discharged to skilled nursing or intermediate care facilities $(18.6 \%$ vs $21.5 \%)$ than hospitals without a JC-certified PSC.

The national RSMR was $10.9 \% \pm 1.7 \%$, with a weighted mean of $10.7 \% \pm 1.7 \%$ for hospitals with a JC-certified PSC (range 6.9\%-16.8\%) and 11.0\% \pm $1.7 \%$ for hospitals without a JC-certified PSC (range $6.2 \%-18.4 \% ; p<0.0001$ for difference). The national RSRR was $12.5 \% \pm 1.4 \%$, and rates were similar between hospitals with and without a JC-certified PSC $(12.5 \% \pm 1.3 \%$ vs $12.4 \% \pm 1.7 \%, p<0.0001$; range $9.2 \%-19.2 \%$ for hospitals with and $8.8 \%-18.4 \%$ for hospitals without JC-certified PSCs). Despite relatively small absolute differences in RSMR and RSRR values between the 2 groups, there was heterogeneity in the distribution of RSMR and RSRR for certified and noncertified hospitals (figure 1).

Almost half of the hospitals with JC-certified PSCs had RSMRs lower than the national average, $45.4 \%$ had rates that were no different, and $5.7 \%$ had higher rates (figure 2). In comparison, only $18.5 \%$ of noncertified hospitals had RSMRs lower than the national average, $66.9 \%$ had rates that were no different, and $14.6 \%$ had risk-standardized rates higher than the national average. The RSRRs were similar between the 2 groups: approximately $13.3 \%$ of hospitals with JC-certified PSCs had RSRRs lower than the national average, as compared with only $15.1 \%$ of noncertified hospitals; $28.3 \%$ of JCcertified PSC hospitals and $23.7 \%$ of noncertified hospitals had 30-day RSRRs higher than the national average. The analyses were repeated using the more restrictive case definition (limiting the sample to those cases with the .X1 modifier). The RSMR and RSRR values increased for this restricted population; however, differences in the RSMR between JCcertified PSC and noncertified hospitals were reduced $(14.7 \% \pm 2.0 \%$ for JC-certified PSC hospitals vs $15.0 \% \pm 1.9 \%$ for noncertified hospitals). Approximately $21.6 \%$ of JC-certified PSC hospitals had RSMRs less than the national average as compared with $16.5 \%$ of noncertified hospitals. The RSRR values were comparable between groups $(14.1 \% \pm 2.1 \%$ in the JC-certified PSC hospitals vs $14.1 \% \pm 1.5 \%$ for the noncertified hospitals), with $12.4 \%$ of JC-certified PSC hospitals having an RSRR less than the national average as compared with $13.8 \%$ of noncertified hospitals.

DISCUSSION Although the absolute differences were small, a higher proportion of hospitals with a JC-certified PSC had 30-day RSMRs that were lower than the national average as compared with hospitals without a JC-certified PSC, but 30-day RSRRs were similar regardless of JC-certified PSC status. There was heterogeneity in the distribution of risk-standardized outcomes for hospitals, with considerable overlap between these groups. The data suggest that JC PSC certification identifies a large number of high-performing hospitals for mortality outcomes, but does not necessarily guarantee better performance than may be found in hospitals without a JC-certified PSC. Moreover, the lack of certification does not necessarily indicate poorer hospital performance.

The majority of prior studies that have evaluated the impact of organized stroke care have focused on mortality and did not assess readmission rates or compare hospital-level outcomes. Similar to our findings, studies conducted outside of the United States have demonstrated that organized stroke care is associated with 
Figure 1 Frequency distribution of 30-day risk-standardized mortality rate (RSMR) and risk-standardized readmission rate (RSRR) by Joint Commission (JC)-certified primary stroke center (PSC) status

A
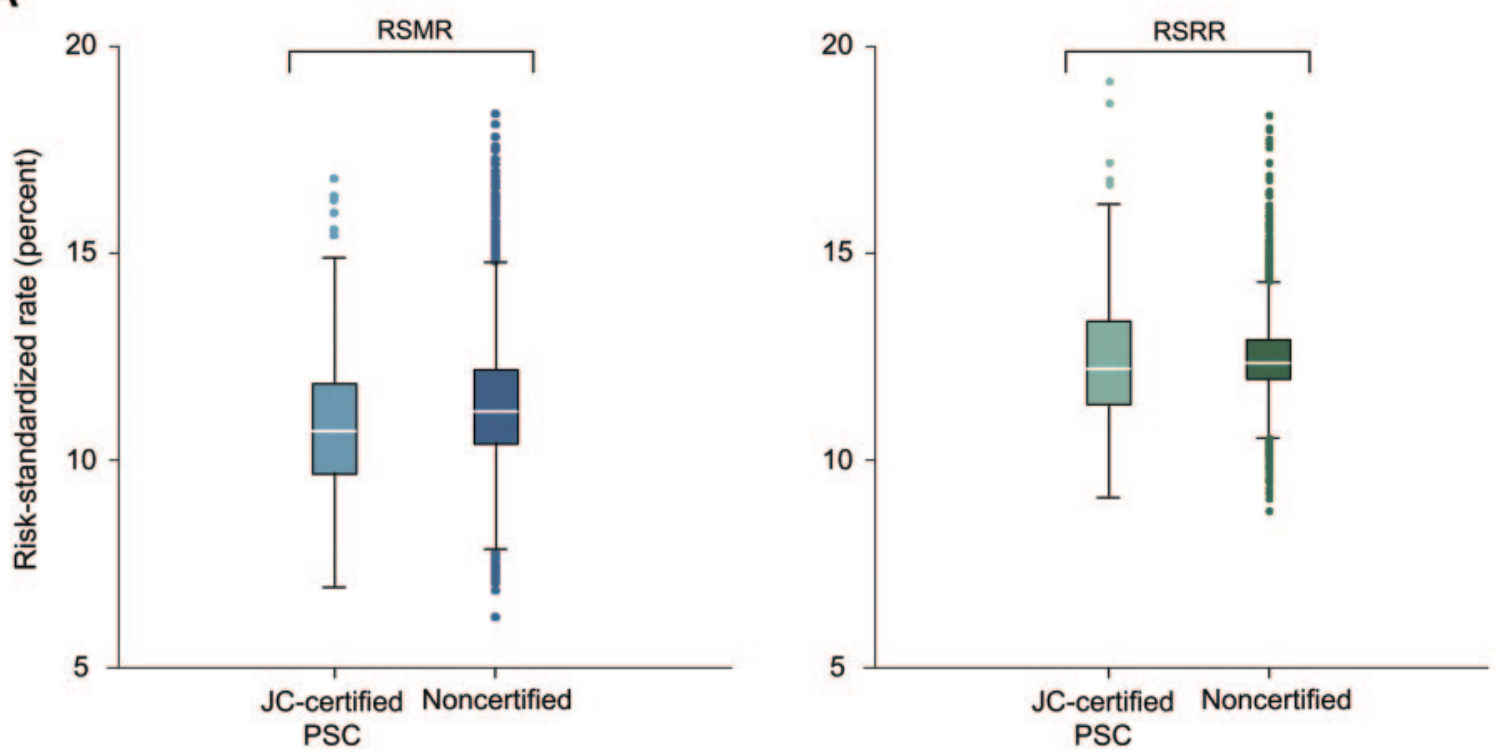

B
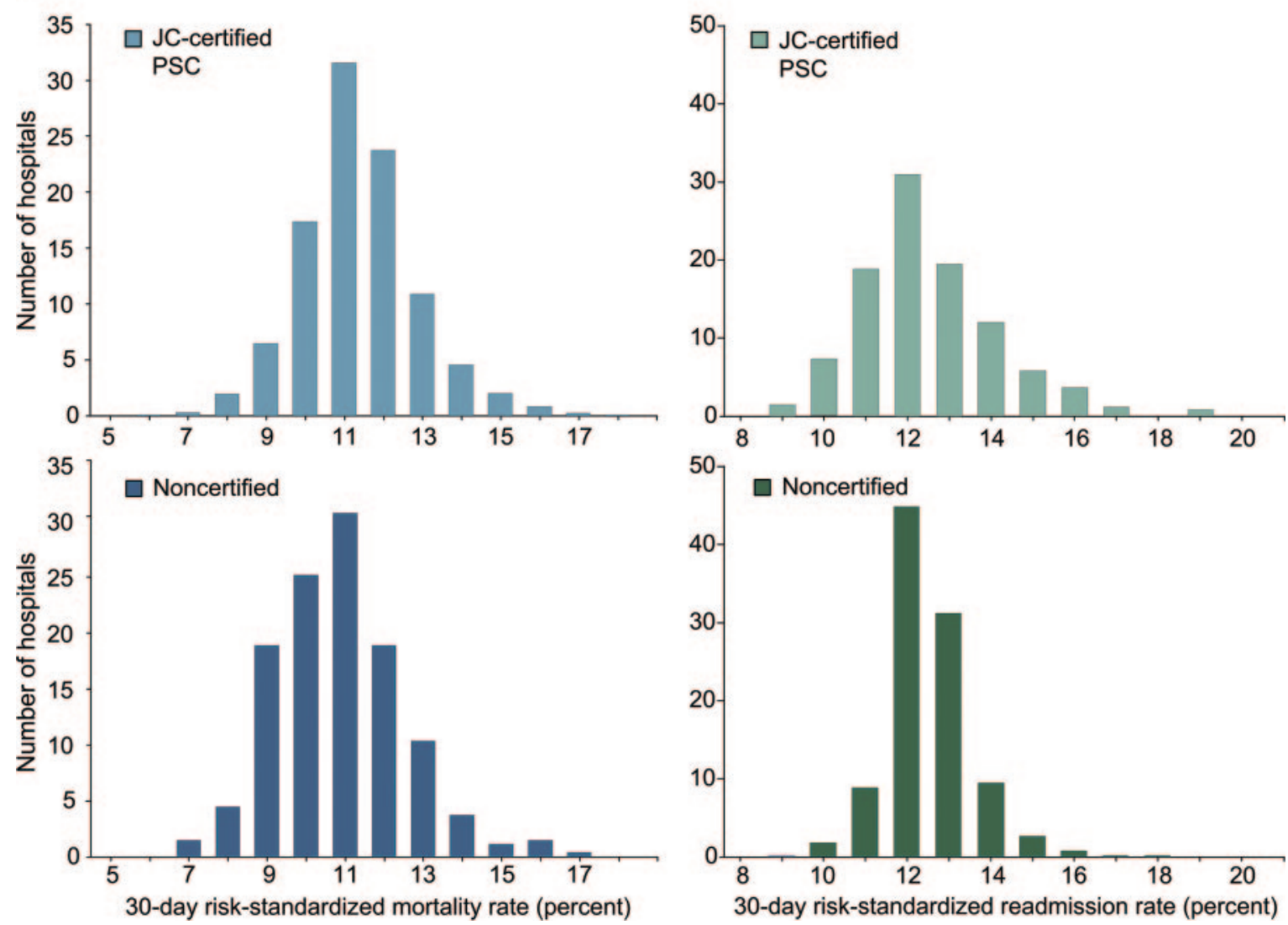

(A) Box and whisker plots of RSMR and RSRR by JC-certified PSC status. The upper boundaries of the boxes represent the 25th percentile, the lines bisecting the boxes represent the median or 50th percentile, and the lower boundaries of the boxes represent the 75th percentile. The lower and upper boundaries of the whiskers are set at the 5 th and 95 th percentiles, with dots representing hospitals beyond these percentiles. (B) Frequency distributions of RSMR and RSRR by JC-certified PSC status.

lower mortality, although there have been inconsistent results as to whether patients uniformly benefit from organized care based on stroke type. ${ }^{25-28}$ One study found that stroke units improve the outcome in patients with large-vessel infarcts, but not in those with lacunar syndromes. ${ }^{28}$ Analyses of organized stroke care in Can- 
Figure 2 Categorization of hospital-level risk-standardized mortality rate (RSMR) and risk-standardized readmission rate (RSRR) relative to the national average by Joint Commission (JC)-certified primary stroke center (PSC) status

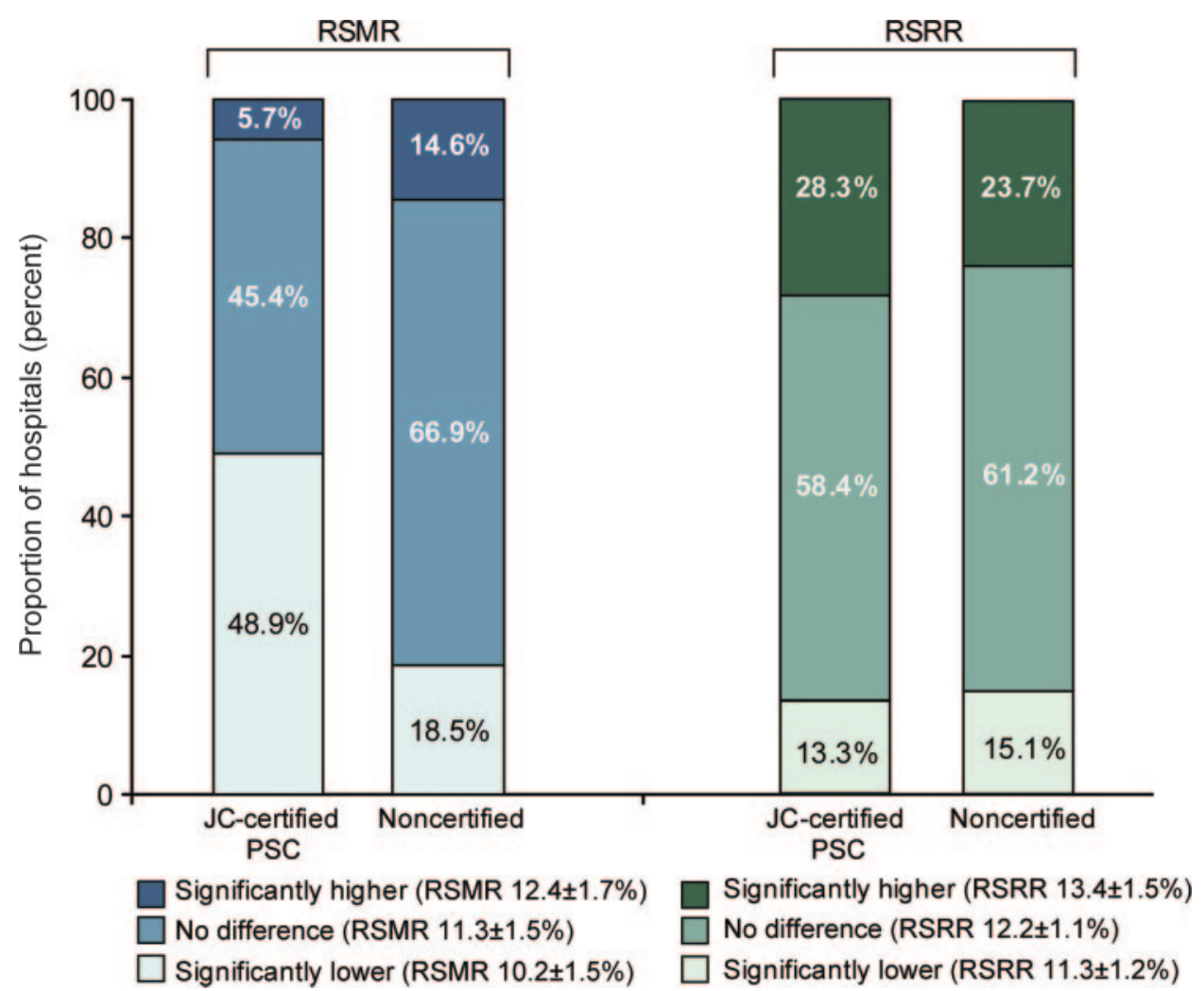

Stacked bars represent the proportions of hospitals significantly higher than (dark blue/green), no different from (medium blue/ green), or significantly lower than (light blue/green) the national RSMR and RSRR, stratified by JC-certified PSC status.

ada show that all age groups and stroke subtypes benefit from organized care, even after adjusting for stroke severity. ${ }^{26,27}$ Additional work found that an increasing level of organized care was associated with improved survival after stroke. ${ }^{25}$ There was heterogeneity in hospital performance within certification categories as well as overlap between JC-certified PSC and noncertified hospitals. This may be partially explained by the variation in the comprehensiveness of care provided within these facilities. In addition, there may be unmeasured factors that contribute to heterogeneity in outcomes. The JCcertified PSC status may be a proxy measure for the resources available at a given facility which serves as an overall indicator of quality of hospital care, but may not adequately measure the variability in the comprehensiveness of care provided.

The present study has several potential limitations. The index ischemic stroke cases were identified using ICD-9-CM codes, and miscoding can occur. Positive predictive values for the selected codes for ischemic stroke, however, are relatively high, ${ }^{23,24}$ and there is no reason to suspect differences in data coding across institutions by JC PSC certification status. Medicare inpatient data do not contain information on stroke severity, an important predictor of out- come. Although discharge location and length of stay at least partially reflect stroke severity, these outcomes are also affected by quality of care. Their inclusion in risk-adjusted models, as well as receipt of therapies such as tissue plasminogen activator use, would therefore confound the analyses. Not adjusting for factors that reflect hospital-level care is consistent with other NQF-endorsed measures used to compare hospital performance. Although studies show that the benefits of organized stroke care do not differ by age group or stroke severity, ${ }^{26}$ there may be variations in referral patterns to facilities. For example, depending on local referral patterns and service availability, moribund patients might be more likely to be kept at local facilities whereas patients with acute symptoms, who may be amenable to thrombolytic therapy, might be preferentially routed to a JCcertified PSC, contributing to better outcomes. There may be additional unmeasured factors that could explain the observed differences in outcomes. Finally, because our analyses are limited to FFS Medicare beneficiaries 65 years or older, the results may not be applicable to those without FFS Medicare coverage or to stroke patients younger than age 
65 years, although Medicare FFS patients in this age group represent the majority of ischemic stroke events.

There are more than 4.4 million stroke survivors in the United States, with approximately 795,000 new strokes identified annually. ${ }^{29,30}$ Stroke is also one of the 10 highest contributors to Medicare costs. ${ }^{31}$ Among the elderly, stroke and TIAs are a leading cause of hospitalization. ${ }^{32,33}$ Recurrent events, which occur in 185,000 stroke survivors in the United States each year, are associated with higher mortality rates, greater levels of disability, and increased costs as compared with initial stroke events..$^{34}$ For stroke survivors, significant disability, preventable complications, and discharge to settings with substantial requirements for ongoing care are common. Due to its high prevalence, adverse outcomes, and large economic burden, stroke represents an important condition to target strategies to reduce mortality and avoidable rehospitalizations. Our study provides initial data on how hospital-level outcome measures, potentially reflecting quality of care, differ by JC PSC certification status. The analysis of hospitallevel outcomes after ischemic stroke shows that JC certification of PSCs identifies many highperforming hospitals, but there remains considerable overlap in outcomes between hospitals with and without a JC-certified PSC. Additional research is needed to identify the key determinants of hospital performance, to reduce variability and improve patient outcomes. Risk-standardized stroke mortality and readmission may be used to inform and motivate health care quality improvement efforts.

\section{AUTHOR CONTRIBUTIONS}

Conception and design: J.H.L., S.B.J., Y.W., L.B.G. Acquisition of data: J.H.L. Analysis or interpretation of data: All authors. Drafting the manuscript: J.H.L., S.B.J., L.B.G. Revising the manuscript for important intellectual content: All authors. Statistical analysis: Y.W., S.B.J., E.W. Obtaining funding: J.H.L. Administrative, technical, material support: J.H.L. Supervision: J.H.L.

\section{ACKNOWLEDGMENT}

The Centers for Medicare \& Medicaid Services reviewed and approved the use of its data for this work and approved submission of the manuscript; this approval is based on data use only and does not represent the Centers for Medicare \& Medicaid Services endorsement of or comment on the manuscript content. The project described was supported by grant numbers R01 NS043322-01 and R01 NS043322 (ARRA) from the National Institute for Neurological Disorders and Stroke. The content is solely the responsibility of the authors and does not necessarily represent the official views of the National Institute of Neurological Disorders and Stroke or the NIH.

\section{DISCLOSURE}

Dr. Lichtman, S.B. Jones, Dr. Wang, E. Watanabe, and Dr. LeifheitLimson report no disclosures. Dr. Goldstein serves on a scientific advisory board for Allergan; has received funding for travel and speaker honoraria from Bayer Schering Pharma; serves on the editorial boards of Neurol- ogy ${ }^{\circledR}$, Emergency Medicine, Stroke, Cerebrovascular Diseases, and Circulation: Cardiovascular Quality and Outcomes and as an Associate Editor for Continuum; receives publishing royalties from $U_{p}$ ToDate and Henry Stewart Talks; serves as a consultant for Pfizer Inc, Boehringer Ingelheim, Johnson \& Johnson, and Merck Serono; receives research support to his institution from Pfizer Inc, AGA Medical Corporation, and Abbott; and receives research support from the NIH, the American Heart Association, and El Centro Hispano.

Received September 9, 2010. Accepted in final form December 27, 2010.

\section{REFERENCES}

1. Adams R, Acker J, Alberts M, et al. Recommendations for improving the quality of care through stroke centers and systems: an examination of stroke center identification options: multidisciplinary consensus recommendations from the Advisory Working Group on Stroke Center Identification Options of the American Stroke Association. Stroke 2002;33:e1-e7.

2. Alberts MJ, Hademenos G, Latchaw RE, et al. Recommendations for the establishment of primary stroke centers: Brain Attack Coalition. JAMA 2000;283:3102-3109.

3. Available at: http://www.jointcommission.org/CertificationPrograms/PrimaryStrokeCenters/guide_table_contents.html; http://www.jointcommission.org/JointCommission/Non PlaceholderTemplates/ListCertifiedOrgs.aspx?NRMODE = Published \&NRNODEGUID =\%7bEC300177-10AA4802-870D-5E79A66476D0\%7d\&NRORIGINALURL= $\% 2 \mathrm{fCertificationPrograms} \% 2 \mathrm{fD}$ isease-SpecificCare $\% 2 \mathrm{fDSCO}$ rgs $\% 2$ fdefault $\% 2$ ehtm\&NRCACHEHINT = Guest. Accessed May 30, 2007.

4. Schwamm LH, Pancioli A, Acker JE 3rd, et al. Recommendations for the establishment of stroke systems of care: recommendations from the American Stroke Association's Task Force on the Development of Stroke Systems. Stroke 2005;36:690-703.

5. Gropen TI, Gagliano PJ, Blake CA, et al. Quality improvement in acute stroke: the New York State Stroke Center Designation Project. Neurology 2006;67:88-93.

6. Douglas VC, Tong DC, Gillum LA, et al. Do the Brain Attack Coalition's criteria for stroke centers improve care for ischemic stroke? Neurology 2005;64:422-427.

7. Lattimore SU, Chalela J, Davis L, et al. Impact of establishing a primary stroke center at a community hospital on the use of thrombolytic therapy: the NINDS Suburban Hospital Stroke Center experience. Stroke 2003;34: e55-e 57 .

8. Stradling D, Yu W, Langdorf ML, et al. Stroke care delivery before vs after JCAHO stroke center certification. Neurology 2007;68:469-470.

9. Lichtman JH, Allen NB, Wang Y, Watanabe E, Jones SB, Goldstein LB. Stroke patient outcomes in US hospitals before the start of the Joint Commission Primary Stroke Center certification program. Stroke 2009;40:3574-3579.

10. Keenan PS, Normand S-LT, Lin Z, et al. An administrative claims measure suitable for profiling hospital performance on the basis of 30-day all-cause readmission rates among patients with heart failure. Circ Cardiovasc Qual Outcomes 2008;1:29-37.

11. Krumholz HM, Merrill AR, Schone EM, et al. Patterns of hospital performance in acute myocardial infarction and heart failure 30-day mortality and readmission. Circ Cardiovasc Qual Outcomes 2009;2:407-413.

12. Krumholz HM, Wang Y, Mattera JA, et al. An administrative claims model suitable for profiling hospital perfor- 
mance based on 30-day mortality rates among patients with an acute myocardial infarction. Circulation 2006; 113:1683-1692.

13. Krumholz HM, Wang Y, Mattera JA, et al. An administrative claims model suitable for profiling hospital performance based on 30-day mortality rates among patients with heart failure. Circulation 2006;113:1693-1701.

14. Medicare Payment Advisory Commission. Report to the Congress: Promoting greater efficiency in Medicare. Available at: http://www.medpac.gov/documents/jun07_ EntireReport.pdf. Accessed February 17, 2010.

15. Medicare fact sheet. Available at: http://www.whitehouse. gov/medicarefactsheetfinal/. Accessed April 2, 2010.

16. Centers for Medicare \& Medicaid Services Hospital Payfor-Performance Workgroup. US Department of Health and Human Services Medicare hospital value-based purchasing plan development: issues paper, 1st public listening session, January 17, 2007. Available at: http:// www.cms.gov/acuteinpatientPPS/downloads/hospital_VBP_ plan_issues_paper.pdf. Accessed April 5, 2010.

17. US Department of Health and Human Services. Hospital compare. Available at: http://www.hospitalcompare.hhs. gov. Accessed April 5, 2010.

18. Institute of Medicine. In: Rewarding Provider Performance: Aligning Incentives in Medicare. Washington, DC: National Academy Press; 2007.

19. Lichtman JH, Leifheit-Limson EC, Jones SB, et al. Predictors of hospital readmission after stroke: a systematic review. Stroke 2010;41:2525-2533.

20. Schisterman EF, Whitcomb BW. Use of the Social Security Administration Death Master File for ascertainment of mortality status. Popul Health Metr 2004;2:2.

21. Krumholz HM, Brindis RG, Brush JE, et al. Standards for statistical models used for public reporting of health outcomes: an American Heart Association Scientific Statement from the Quality of Care and Outcomes Research Interdisciplinary Writing Group: cosponsored by the Council on Epidemiology and Prevention and the Stroke Council: endorsed by the American College of Cardiology Foundation. Circulation 2006;113:456-462.

22. Normand S-LT, Wang Y, Krumholz HM. Assessing surrogacy of data sources for institutional comparisons. Health Serv Outcomes Res Method 2007;7:79-96.
23. Goldstein LB. Accuracy of ICD-9-CM coding for the identification of patients with acute ischemic stroke: effect of modifier codes. Stroke 1998;29:1602-1604.

24. Tirschwell DL, Longstreth WT Jr. Validating administrative data in stroke research. Stroke 2002;33:2465-2470.

25. Saposnik G, Fang J, O’Donnell M, Hachinski V, Kapral MK, Hill MD. Escalating levels of access to in-hospital care and stroke mortality. Stroke 2008;39:2522-2530.

26. Saposnik G, Kapral MK, Coutts SB, Fang J, Demchuk AM, Hill MD. Do all age groups benefit from organized inpatient stroke care? Stroke 2009;40:3321-3327.

27. Smith EE, Hassan KA, Fang J, Selchen D, Kapral MK, Saposnik G. Do all ischemic stroke subtypes benefit from organized inpatient stroke care? Neurology 2010;75:456462 .

28. Evans A, Harraf F, Donaldson N, Kalra L. Randomized controlled study of stroke unit care versus stroke team care in different stroke subtypes. Stroke 2002;33:449-455.

29. Lloyd-Jones D, Adams R, Carnethon M, et al. Heart disease and stroke statistics: 2009 update: a report from the American Heart Association Statistics Committee and Stroke Statistics Subcommittee. Circulation 2009;119: $480-486$.

30. Sacco RL, Shi T, Zamanillo MC, Kargman DE. Predictors of mortality and recurrence after hospitalized cerebral infarction in an urban community: The Northern Manhattan Stroke Study. Neurology 1994;44:626-634.

31. Andrews R, Elixhauser A. The National Hospital Bill: Growth Trends and 2005 Update on the Most Expensive Conditions by Payer: Healthcare Cost and Utilization Project: Statistical Brief \#42. Washington, DC: Agency for Healthcare Research and Quality; 2007.

32. Hennen J, Krumholz HM, Radford MJ. Twenty most frequent DRG groups among Medicare inpatients age 65 or older in Connecticut hospitals, fiscal years 1991, 1992, and 1993. Conn Med 1995;59:11-15.

33. DeFrances CJ, Lucas CA, Buie VC, Golosinskiy A. National Hospital Discharge Survey. Natl Health Stat Rep 2008;5:1-20.

34. Samsa GP, Bian J, Lipscomb J, Matchar DB. Epidemiology of recurrent cerebral infarction: a medicare claimsbased comparison of first and recurrent strokes on 2-year survival and cost. Stroke 1999;30:338-349.

\section{Neurology ${ }^{\circledR}$ Launches Subspecialty Alerts by E-mail!}

Customize your online journal experience by signing up for e-mail alerts related to your subspecialty or area of interest. Access this free service by visiting http://www.neurology.org/cgi/alerts/ etoc.xhtml or click on the "E-mail Alerts" link on the home page. An extensive list of subspecialties, methods, and study design choices will be available for you to choose from-allowing you priority alerts to cutting-edge research in your field! 